\title{
Search for Top Quark at CDF
}

\author{
Jaroslav Antos \\ For the CDF Collaboration \\ Fermi National Accelerator Laboratory \\ P.O. Box 500, Batavia, Illinois 60510
}

November 1994

Published Proceedings Hadron Structure '94, Safarik University, Kosice, Slovakia, September 19-23, 1994 


\section{Disclaimer}

This report was prepared as an account of work sponsored by an agency of the United States Government. Neither the United States Government nor any agency thereof, nor any of their employees, makes any warranty, express or implied, or assumes any legal liability or responsibility for the accuracy, completeness, or usefulness of any information, apparatus, product, or process disclosed, or represents that its use would not infringe privately owned rights. Reference herein to any specific commercial product, process, or service by trade name, trademark, manufacturer, or otherwise, does not necessarily constitute or imply its endorsement, recommendation, or favoring by the United States Government or any agency thereof. The views and opinions of authors expressed herein do not necessarily state or reflect those of the United States Government or any agency thereof. 


\section{DISCLAIMER}

Portions of this document may be illegible in electronic image products. Images are produced from the best available original document. 


\title{
Search for Top Quark at CDF
}

\author{
Jaroslav Antoš ${ }^{1}$ \\ for the CDF collaboration
}

\begin{abstract}
Recently CDF collaboration presented evidence for direct observation of t $\bar{t}$ production [1] in $19.3 \mathrm{pb}^{-1}$ of $\mathrm{p} \overline{\mathrm{p}}$ collisions at $\sqrt{(s)}=1.8 \mathrm{TeV}$. Very brief account of these results is presented here.
\end{abstract}

There is vast theoretical and experimental support for idea that top quark as a weak isospin partner to b-quark should exist. So far unsuccessful searches provided us with knowledge about lower limit on its mass. Standard model dependent lower limit on top quark mass was found as high as $131 \mathrm{GeV} / \mathrm{c}^{2}$ [2]. Production cross section is steeply falling function of top quark mass. Therefore realistically at present only Tevatron $p \bar{p}$ collider at FNAL, with total energy $1.8 \mathrm{TeV}$ in CMS system, still has a chance of top quark discovery. Dominant production mechanism for top quarks at Tevatron is pair production of $t \bar{t}$. With almost $100 \%$ probability $t(\bar{t})$ decays in mode $t \rightarrow W^{+} b$. Distinct features of this decay provide very good signatures of top quark production which helps to reduce otherwise very high level of background. Based on simple combinatorial arguments one can show that $W$ should decay in $\frac{1}{9}$ cases into $W \rightarrow l+\nu$ where 1 stands for lepton $(e, \mu, \tau)$. Very clean signature represents case when both W's from $t$ and $\bar{t}$ decay into e $(\mu)+\nu$. In this case experimental observation will be two isolated leptons (in this article as isolated leptons are considered only electrons or muons) characterized by large transverse momentum, large missing transverse energy $\mathbb{E}_{\tau}$ (carried out by 2 neutrinos) and $2 \mathrm{~b}$ quark jets. Jets originated from $\mathrm{b}$ quarks can be quite frequently recognized by presence of secondary vertices associated with jets (coming from decay of hadrons with $b$

${ }^{1}$ present address: Institute of Physics, Academia Sinica, Taiwan

Published Proceedings Hadron Structure '94, Safarik University, Kosice, Slovakia, September 19-23, 1994. 
(c) quark component). Another feature of b-jets which can be used for their identification is frequent association of so called soft leptons (electrons or muons with $\mathrm{P}_{\mathrm{T}}>2 . \mathrm{GeV} / \mathrm{c}$ ) with jets.

Somewhat larger background but hopefully manageable can be expected in case when one of W's decays in lepton mode and other into two quarks. Therefore ideal detector for top quark search should meet following objectives:

- lepton identification

- jet reconstruction

- hermiticity (good $\mathbb{E}_{T}$ resolution)

- b-jet identification

Two experimental setups CDF and DO are able to take advantage of Tevatron for top quark discovery. These two setups are build in sort of complementary way. CDF with emphasis on excellent tracking in central region provided by central tracking chamber (CTC) inside uniform magnetic field of intensity $1.4 \mathrm{~T}$ produced by superconducting solenoid and very good vertexing given by silicon vertex detector (SVX). Full description of CDF setup is given in [3]. D0 relies more on calorimetry, hermeticity of design (better $\mathbb{E}_{\mathrm{T}}$ resolution). Description of D0 can be found in [4].

\section{Search for $t \bar{t}$ events decaying in dilepton mode}

As discussed above signal from $t \bar{t}$ events in this mode will be two isolated leptons with $\mathrm{P}_{\mathrm{T}}>20 \mathrm{GeV} / \mathrm{c}$ each, at least two jets in an event and $\mathbb{E}_{\mathrm{T}}>25 \mathrm{GeV}$. Leptons should have opposite sign and to suppress background coming from $Z^{0}$ production, pairs of the same flavor with invariant mass between $75-105 \mathrm{GeV} / \mathrm{c}^{2}$ are excluded from top candidate sample. In case when azimuthal angle between lepton (jet) is less than 20 degrees, cut on missing transverse energy of $t \bar{t}$ candidates is risen to $50 \mathrm{GeV}$. Justification of above cuts can be seen from fig. 1 from Monte Carlo simulation by isajet [5] for top quark mass hypothesis $160 \mathrm{GeV} / \mathrm{c}^{2}$ and from Drell-Yan data sample on fig.2.

Statistics of survived data after sequential application of cuts is presented in tab.1. As a results of all cuts applied no dilepton events of the same flavor survived. Two e $\mu$ events passed all cuts.

Estimate of level of different kind of background passing the same selection criteria is given in tab.2. One can see that on average background is expected to be on level of 0.56 events to observed 2 !

\section{Search for $t \bar{t}$ candidates in events with isolated lepton + jets}

To beat significantly larger background then in previous case we require at least 3 jets with $\mathrm{P}_{\mathrm{T}}>15 \mathrm{GeV} / \mathrm{c}$ to accompany isolated lepton and $\mathbb{E}_{\mathrm{T}}>20 \mathrm{GeV}$ to consider this event 


\begin{tabular}{lccc}
\hline \hline \multicolumn{1}{c}{ Cut } & $e \mu$ & $e e$ & $\mu \mu$ \\
\hline $\mathrm{P}_{\mathrm{T}}$ & 8 & 702 & 588 \\
Opposite-Charge & 6 & 695 & 583 \\
Isolation & 5 & 685 & 571 \\
Invariant Mass & 5 & 58 & 62 \\
$\mathrm{E}_{\mathrm{T}}$ magnitude & 2 & 0 & 1 \\
$\mathrm{E}_{\mathrm{T}}$ direction & 2 & 0 & 0 \\
Two-jet & 2 & 0 & 0 \\
\hline \hline
\end{tabular}

Table 1: Number of data events surviving consecutive requirements.

\begin{tabular}{llc}
\hline \hline & & All cuts \\
\hline$e \mu$ & $W W$ & $0.10 \pm 0.04$ \\
$Z \rightarrow \tau \tau$ & $0.07 \pm 0.02$ \\
& $b \bar{b}$ & $0.04 \pm 0.03$ \\
& Fake & $0.03 \pm 0.03$ \\
& & \\
& Total background & $0.24 \pm 0.06$ \\
& CDF data & 2 \\
ee, $\mu \mu$ & $0.06 \pm 0.02$ \\
& $Z \rightarrow \tau \tau$ & $0.06 \pm 0.02$ \\
& $b \bar{b}$ & $0.05 \pm 0.03$ \\
& Fake & $0.04 \pm 0.03$ \\
& Drell-Yan & $0.10_{-0.08}^{+0.23}$ \\
& Total background & $0.31_{-0.10}^{+0.24}$ \\
& CDF data & 0 \\
\hline \hline
\end{tabular}

Table 2: Number of background events expected in $19.3 \mathrm{pb}^{-1}$ and the number of events observed in the data. 
as $t \bar{t}$ candidate. One can see effect of different cuts on data sample in tab.3. and statistics of final sample as a function of jet multiplicity in tab.4 There are 52 events with $\geq 3$ jets surviving all kinematical and quality cuts. Background to these events can be divided into two categories:

1. QCD jets, WW,ZZ,WZ production and $\mathrm{Z} \rightarrow 11$

2. $W+$ jets

First category of background was carefully studied and was estimated to account for $11.9 \pm 3.1$ events.

Second category was studied using vecbos model [6]. Comparison of VECBOS model calculation and data are given in tab.j.

One can see that result from model is very close to observed number of events. But it is known that there is a factor 2 theoretical uncertainty in calculation of scale of cross section. In this case one should more carefully examin 52 candidate events to determine more precisely their origin. In $t \bar{t}$ events there should be 2 b-jets present in each event and therefore ability to distinguish b-jets provides handle to origin of these events.

\section{b-tagging}

Secondary vertex (inconsistent with $\Lambda, K_{s}, \gamma$ conversion) associated with one of selected jets provides evidence for heavy quark jet. This hypothesis can be tested on data samples with known b-jet content, one can compare it with estimate based on vertexing. Another independent test is to look at $c \tau$ distribution (which can be obtained when all particles in secondary vertex are fully reconstructed, identified - based on Monte Carlo studies one can argue that reasonable $c T$ estimate can be obtained even if these conditions are not fully satisfied). B-tagging algorithm called JETVTX satisfactory passed both tests. Basically it takes advantage of very good SVX resolution, requires at least two good three dimensional tracks reconstructed in SVX with significantly large impact parameters relatively to primary vertex, and then attempts to reconstruct common vertex from selected tracks is made.

Based on Monte Carlo studies it was found that this algorithm is about $22 \%$ efficient for $t \bar{t}$ events almost independent of top quark mass in mass range $130-180 \mathrm{GeV} / \mathrm{c}^{2}$. Applying this algorithm to our 52 event sample 6 events have been tagged. Once again very important question is how many tags one expects in case when all 52 events come from background. To make this estimate one parametrize tagging rate for generic (unbiased) jets sample as a function of transverse energy, track multiplicity in jet, number of jets in event. Applying this parametrisation and assumption that tagging rate in generic jets is the same as corresponding tagging rate in jets accompanying $W$ (this assumption is considered to be conservative, because in generic jets should be larger fraction of heavy 


\begin{tabular}{lcc}
\hline \hline Selection Criteria & Electrons & Muons \\
\hline Good Lepton & 28,522 & 17,994 \\
Lepton Isolation Requirement & 20,420 & 11,901 \\
$Z$ Removal & 18,700 & 11,310 \\
$\mathbb{E}_{\mathrm{T}}>20 \mathrm{GeV}$ & 13,657 & 8,724 \\
Good Quality Run & $12,79.7$ & 8,272 \\
Trigger Requirement & 11,949 & 7,024 \\
\hline \hline
\end{tabular}

Table 3: The number of events passing various consecutive selection criteria in data. The good lepton requirement includes all quality selection, fiducial requirements, $E_{\mathrm{T}}$ cuts, and conversion removal.

\begin{tabular}{lcc}
\hline \hline Jet Multiplicity & Electron Events & Muon Events \\
\hline 0 Jet & 10,663 & 6,264 \\
1 Jet & 1058 & 655 \\
2 Jets & 191 & 90 \\
3 Jets & 30 & 13 \\
$\geq$ 4 Jets & 7 & 2 \\
\hline \hline
\end{tabular}

Table 4: Jet multiplicity dependence of final sample of isolated leptons. 
quark component then in jets accompanying $W$ ) to jets of our 52 events sample number of tagged events is $\approx 2$. Taking into account also 1 . category background and if we assume that all $j 2$ events come just from background one can expect to tag on average only $2.3 \pm 0.29$ events.

Another tagging method (SLT) is based on detection of soft lepton associated with jet. By this method 7 events have been tagged and $3.1 \pm 0.3$ tags would be expected if background is only source. Three events have been tagged by both taggers. These events have much smaller probability to be just from background then others! Comparison of number of tags as a function of number of jets in data with expectated background is given on fig.4

\section{Statistical significance of the results}

What is probability that known background fluctuated to observed number of $t \bar{t}$ candidate events? For dilepton signal probability to observe at least two events purely from background was found to be $12 \%$. For SVX b-tagged events probability that they all come from background was found to be $3 \%$ and for soft lepton tagged events $=\% \%$. Combined estimate correctly taking into account correlation between numbers of events tagged by different methods gives probability $0.26 \%$ that observed ti candidates are all just fluctuation of background.

\section{Measurement of top quark mass}

For top mass reconstruction in lepton + jets events (tagged by SVX or SLT method) it is necessary to have in each event at least 4 jets. These events have been reanalyzed and for 4 -th jet more relaxed criteria have been used. In 7 from 10 events really 4 -th jet was found. Then kinematical constraints on production and decay of $t \bar{t}$ in $p \bar{p}$ collision at $1800 \mathrm{GeV}$ have been used to fit top quark mass. In fit mass of $\mathrm{W}$ was fixed and both top quarks in the event have been constraint to have the same mass. There are ambiguities in assignment of correct combination of 3 jets or 2 jets + lepton to common $t(\overline{\mathrm{t}})$ vertex. In this case combination which results in lower $\chi^{2}$ of constraint fit have been choosen. Given procedure was tested on Monte Carlo events. Peak of reconstructed top quark mass is very close to original parameter set in simulation (see fig.5). Naturally distribution is much broader than the same reconstructed mass distribution when alweys correct choice in case of ambiguities was made. Reconstructed top quark mass for these events is in tab.6.

Most probable top quark mass was calculated by simultaneous fit of likelihood function in which background from $\mathrm{W}+\mathrm{jets}$ and $t \bar{t}$ signal are incorporated. Logarithm of likelihood dependence on top quark mass is presented on fig.6. Most probable top quark mass was found to be $174 \pm 10_{-12}^{+13} \mathrm{GeV} / \mathrm{c}^{2}$. Because acceptance of $t \bar{t}$ events dependends 


\begin{tabular}{lcc}
\hline \hline Jet Multiplicity & Data & VECBOS $\left(Q^{2}=\left\langle\mathrm{P}_{\mathrm{T}}\right\rangle^{2}\right)$ \\
\hline 1 Jet & 1713 & $1571 \pm 82_{204}^{+267} \pm 55$ \\
2 Jets & 281 & $267 \pm 20_{-53}^{+77} \pm 9$ \\
3 Jets & 43 & $39 \pm 3_{-3}^{+11} \pm 2$ \\
$\geq 4$ Jets & 9 & $7 \pm 1_{-2}^{+3} \pm 0.2$ \\
\hline \hline
\end{tabular}

Table 5: Comparison of $W+$ jet(s) yields with expectations from the VECBOS Monte Carlo program. The first uncertainty on the VECBOS prediction is due to Monte Carlo statistics, the second is due to jet energy scale and lepton identification efficiency uncertainties, the third is due to the luminosity normalization. The VECBOS predictions include the $W \rightarrow \tau \nu$ contribution. The data have not been corrected for 1 . cathegory backgrounds discussed in the text.

\begin{tabular}{cclcc}
\hline \hline Nev & $\ell$ & $\begin{array}{l}\text { b-tag } \\
\text { b-tag }\end{array}$ & $\begin{array}{c}\text { mass } \\
\left(\mathrm{GeV} / \mathrm{c}^{2}\right)\end{array}$ & $\chi^{2}$ \\
\hline 1 & $e^{+}$ & $\operatorname{SVX}$ & $172 \pm 11$ & 0.0 \\
2 & $e^{-}$ & $\operatorname{SVX}$ & $166 \pm 11$ & 2.0 \\
3 & $\mu^{-}$ & $\operatorname{SLT}\left(\mu^{-}\right)$ & $158 \pm 18$ & 6.1 \\
4 & $\mu^{+}$ & $\operatorname{SVX}$ & $180 \pm 9$ & 5.0 \\
5 & $e^{-}$ & $\operatorname{SLT}\left(e^{+}\right)$ & $188 \pm 19$ & 0.4 \\
6 & $\mu^{+}$ & $\operatorname{SLT}\left(\mu^{-}\right), \operatorname{SVX}$ & $169 \pm 10$ & 2.2 \\
7 & $e^{-}$ & $\operatorname{SLT}\left(e^{-}\right)$ & $132 \pm 8$ & 1.7 \\
\hline \hline
\end{tabular}

Table 6: Characteristics of the seven candidate events: run and event number, lepton type, tagging algorithm (including sign of lepton for the SLT tagging algorithm), top combination that includes the tagged $b$, fitted mass and its uncertainty, and the $\chi^{2}$ from the fit. 
on top quark mass, also does estimate of production cross section. If we use as input our

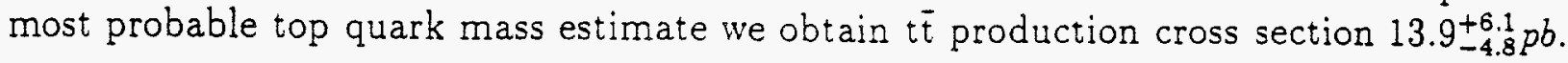

\section{Summary of D0 results [7]}

D0 found 1 t $\bar{t}$ candidate in dilepton search mode $(e \mu)$ on background level $0 . \dot{8}$ and 6 candidates in lepton + jets search mode. Therefore it totally collected 7 candidates on background level of 3.2 They concluded that their results are compatible with both CDF results or just with background.

\section{LEP EW working group results}

Very impressive analysis was presented at this conference from LEP electroweak working group see [8]. Indirect estimate of top quark mass from global analysis of electroweak precision measurements gives value $178 \mathrm{GeV} / \mathrm{c}^{2}$ which is very close to CDF direct measurement.

\section{Conclusion:}

CDF presents evidence for detection of $t \bar{t}$ events, but because of small statistics this can not be considered as a proof. Three independent analysis (concerning t $\bar{t}$ production and properties) CDF,D0 and LEP electroweak working group are compatible.

I would like to thank organizers of this conference for their effort which resulted in very frendly and stimulating atmosphere there.

I would like also to thank my colleagues at FNAL and in Taiwan specially to G.P. Yeh, S.C. Lee and Y.C. Chen. Communication with them and their criticism helped very much to my understanding of the subject. 


\section{References}

[1] F. Abe et al., Fermilab-Pub-94/097-E, accepted to Phys. Rev.D

F. Abe et al., Phys. Rev. Lett. 73 (1994)225

[2] S. Abachi et al., Phys. Rev. Lett. 72 (1994)2138

[3] F. Abe et al., Nucl. Inst. Meth. A271 (1988)387

D. Amidei et al., Fermilab-Pub-94/024-E, submitted to Nucl. Ins. Meth.

[4] S. Abachi et al., Nucl. Inst. Meth. A324 (1993)53

[5] F. Paige and S.D. Protopopescu, BNL Report \o38034, 1986

[6] F.A. Berends, W.T. Giele, H. Kuijf and B. Tausk, Nucl. Phys. B357, (1991)32

[7] P. Grannis, contribution to ICHEP, Glasgow,20-27th July 1994

[8] B.Vallage - contribution to this conference 

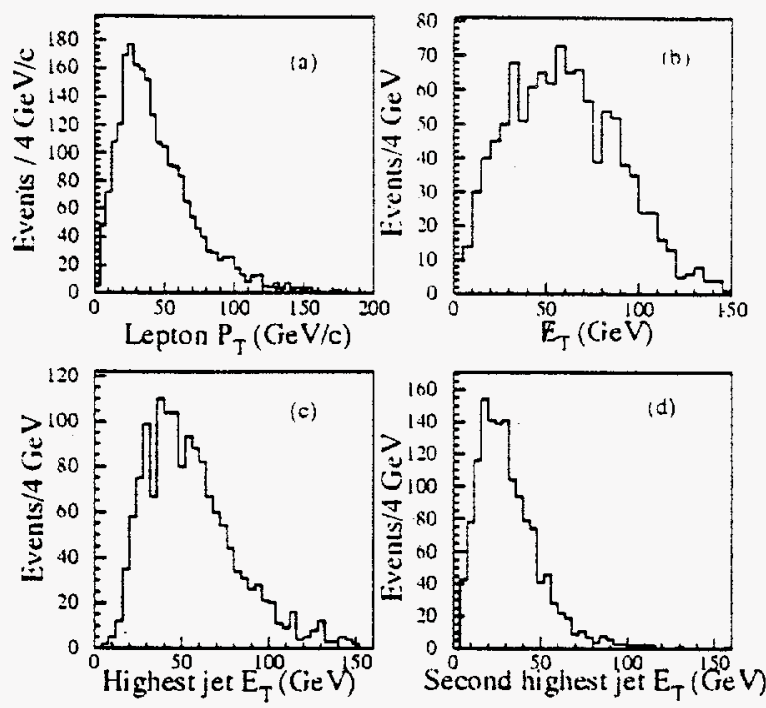

Figure 1: Monte Carlo distributions for $M_{\text {top }}=160 \mathrm{GeV} / \mathrm{c}^{2}$. a) Lepton $\mathrm{P}_{\mathrm{T}}$ spectrum from $t \rightarrow W \rightarrow l \mathrm{~b}) \mathbb{E}_{\mathrm{T}}$ for events with two leptons with $\mathrm{P}_{\mathrm{T}}>20 \mathrm{GeV} / \mathrm{c}$ d) Next to leading jet $E_{\mathrm{T}}$ for dilepton events
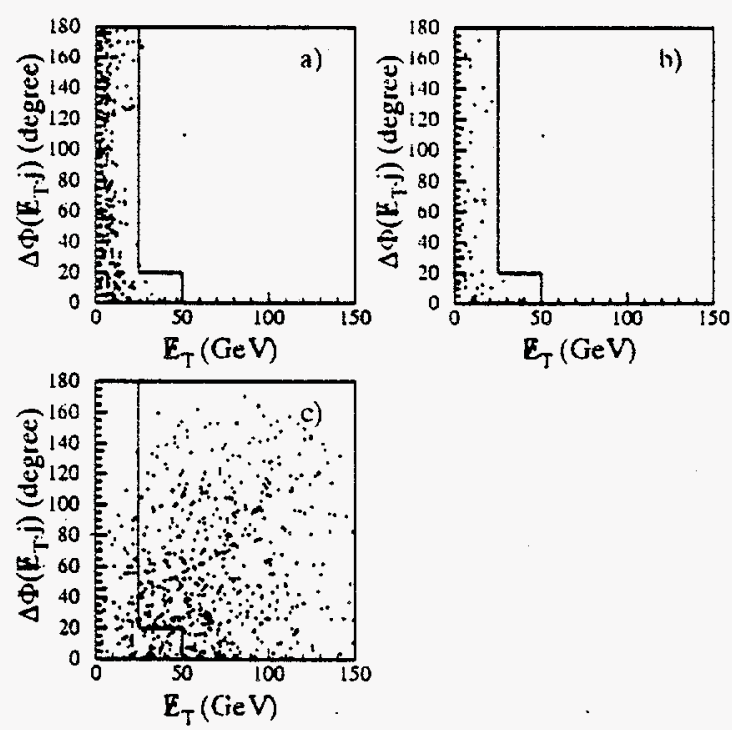

Figure 2: Distribution of azimuthal angle between $\mathbb{E}_{\mathrm{T}}$ and the closest jet versus $\mathbb{E}_{\mathrm{T}} \mathrm{a}$ ) Dielectron and dimuon data from a Drel-Yan control sample $Z^{0}+\geq 1$ jet events. b) Same for sample of $Z^{0}+\geq 2$ jet events. c) Monte Carlo simulation for $M_{\text {top }}=160 \mathrm{GeV} / \mathrm{c}^{2}$ (unnormalized). The boundary defines signal region. 

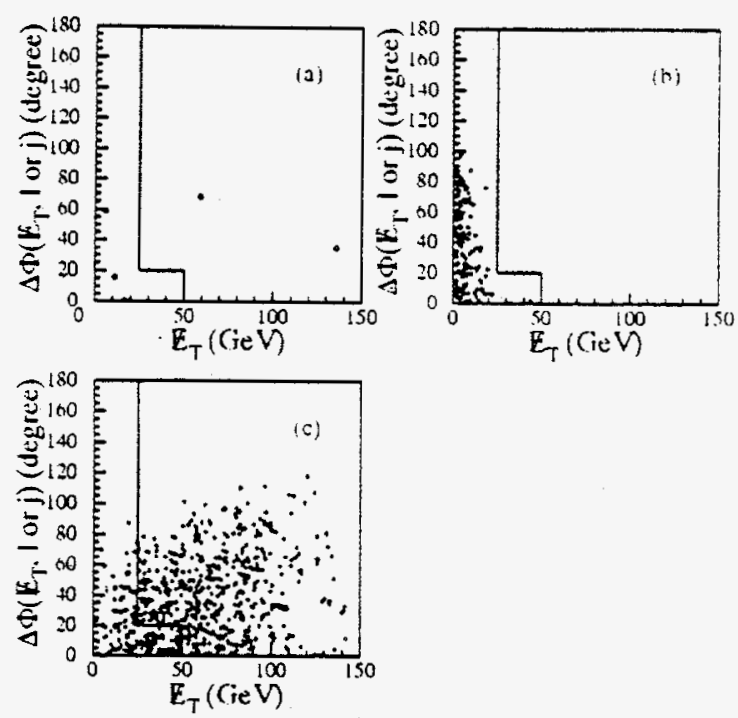

Figure 3: Distribution of azimuthal angle between $\mathbb{E}_{\tau}$ and the closest lepton or jet versus $\mathbb{Z}_{\mathrm{r}}$ a) $e \mu$ b) Dielectron and dimuon data after invariant mass cut. c) Monte Carlo simulation for $M_{\text {top }}=160 \mathrm{GeV} / \mathrm{c}^{2}$ (unnormalized).

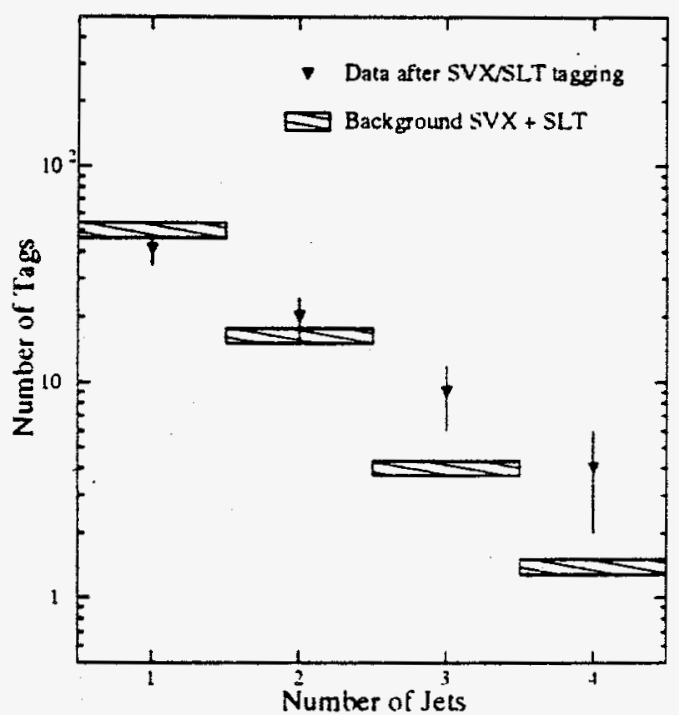

Figure 4: The sum of SVX and SLT tags observed in $\mathrm{W}+$ jets data (solid triangles). Events tagged by both algorithms are counted twice. The shaded area is the sum of the background estimates for SVX and SLT, with its error. 


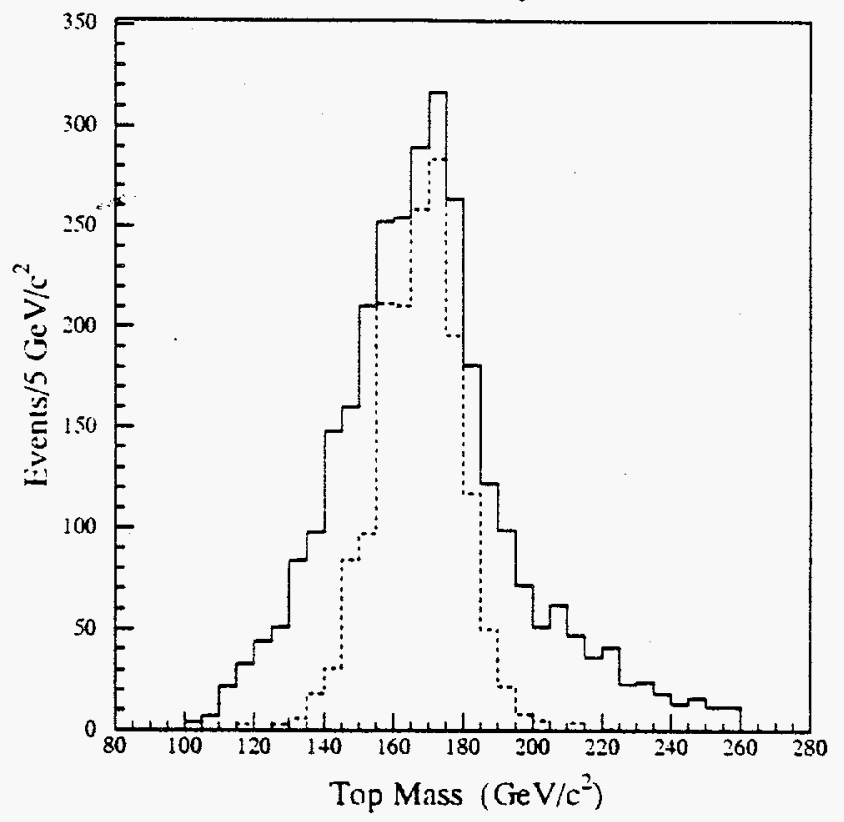

Figure 5: Reconstructed top mass distribution for Monte Carlo events generated with $M_{\text {top }}=170 \mathrm{GeV} / \mathrm{c}^{2}$. The full histogram corresponds to best fit obtained by fitting program following selection procedure. The dashed histogram refers to the fit with correct assignment for each of the jets.

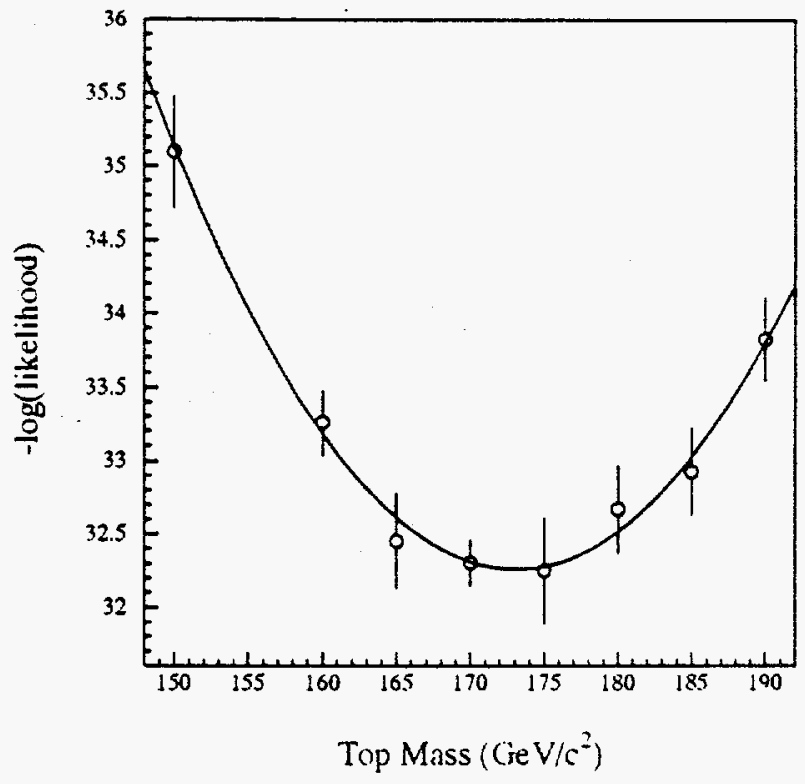

Figure 6: Likelihood fit of top mass 